\title{
Use of orthospin technology in the correction of complex limb deformities
}

Received: 05 August, 2021

Accepted: 17 August, 2021

Published: 18 August, 2021

*Corresponding author: Adam D Geffner, Department of Limb Lengthening and Complex Reconstruction, Hospital for Special Surgery, New York, United States, E-mail: geffnera@hss.edu, adamgeffner@yahoo.com https://www.peertechzpublications.com

\section{Check for updates}

\section{Adam D Geffner*, Taylor J Reif, Austin T Fragomen and S Robert Rozbruch}

Department of Limb Lengthening and Complex Reconstruction, Hospital for Special Surgery, New York, United States

\begin{abstract}
Circular external fixators using hexapod struts are a valuable tool in the treatment of complex, multiplanar limb deformities. The benefits of external fixation technology are well documented and include excellent biomechanical fixation allowing weight bearing and range of motion of joints during treatment, as well as superb versatility to manage a host of challenging clinical pathologies. Despite these advantages, proper treatment with a hexapod external fixation device depends on the patient's ability to manually adjust the six struts according to a demanding treatment plan. Without strict adherence to the treatment plan, prolonged treatment, worsening deformity, or even the need for further surgery become possible. Orthospin's Autostrut technology provides a solution to the drawbacks of external fixation treatment by automating the strut-adjustment process. A control box automatically turns motorized struts according to the physician's treatment plan and sends real-time updates to the physician's phone or computer. This allows him or her to closely monitor the treatment process and make necessary adjustments as needed. Orthospin technology is FDA approved and given its success in early applications is ready for more widespread use.
\end{abstract}

\section{Introduction}

External fixation devices are often the preferred method of treatment for complex limb deformities due to their ability to sequentially or simultaneously correct multiplanar deformities. They can be applied to any long bone or joint deformity and are extremely versatile, allowing multiple simultaneous deformity corrections on a single limb [1]. External fixators often provide superior biomechanical properties compared with other orthopedic devices like plate and screw or intramedullary nail constructs [2]. The original Ilizarov circular ring fixators developed in the 1950 s used thin, highly tensioned wires to hold and manipulate bone fragments along threaded rods [3]. The Taylor Spatial Frame (TSF) and many subsequent external fixation systems introduced hexapod strut technology [2] which utilizes gradual lengthening or shortening of six telescopic struts to correct limb deformities along any angular or translational plane ("6 axes of freedom") with the help of a computer-generated adjustment schedule [5]. The physician is thus able to precisely control the length and alignment of a limb via incremental strut adjustments [4]. Any residual deformity is managed with another shorter application of the process.

The success of external fixators in the correction of complex limb deformities has been well documented. A 2008 study analyzed 38 patients with a tibial nonunion treated with a hexapod circular fixator. Bony union was achieved after the initial treatment in $27(71 \%)$ patients, and after an additional treatment schedule, 36 (95\%) ultimately experienced bony union [6]. A 2010 study of 122 tibial deformity patients treated with hexapod technology demonstrated all tibial deformities were corrected accurately and with few complications [7]. Similarly, a retrospective review of 52 patients who underwent supramalleolar tibial osteotomy with application of a hexapod fixator found significant improvement in all aggregate postoperative distal tibial angles [8].

While the results of these studies exemplify the remarkable effectiveness and dynamic nature of external fixators, there are 
drawbacks to the process worth noting. When the patient leaves the hospital after external fixator application, he or she is given a piece of paper that outlines how often, by how much, and in what direction to adjust the hexapod struts [9]. Successful treatment relies on strict adherence to this guide, and it is the responsibility of the patient to manually adjust the struts [9]. In some cases, patients are unable to adjust their own struts, and must have a caregiver who can frequently, consistently, and accurately turn the struts in accordance with the treatment plan. The patient or caregiver must turn as many as 6 struts up to 4 times a day, equating to over 2100 turns during a 3-month treatment plan. This leaves many opportunities for patient error [10]. In the experience of the senior author, around $10 \%$ of patients experience problems associated with manual strut adjustments, particularly among those with poor fine motor coordination or eyesight, or those who are lackadaisical with strict scheduled regimens. Additionally, since doctors cannot monitor compliance with the treatment plan, and unrecognized errors prolong the period of adjustments, the patient needs transportation to attend frequent office visits. While difficult to quantify for each patient, transportation costs and lost days of work for the patient or the caregiver could increase the cost of treatment by thousands of dollars. If a patient cannot meet all of these requirements, treatment with hexapod external fixator may not be viable, and the patient will not take advantage of the benefits associated with external fixation [1].

\section{Orthospin technology}

Recent developments in robotics have provided a solution to the deficiencies of external fixation treatment. Orthospin, located in Yoqneam, Israel, has developed a smart robotic system for external fixation devices. Their AutoStrut technology involves 6 motorized "Smartstruts" that receive information from a smart control box placed on top of the external fixator frame (Figure $1 \mathrm{~A}$ and $1 \mathrm{~B}$ ) [10]. Orthospin's system automatically and accurately adjusts and lengthens struts in accordance with the physician's prescribed treatment plan. This process is fully automated, and eliminates the dependency on patient compliance and the need for manual strut adjustments [11]. The adjustments can theoretically occur up to 100 times per day, which should decrease the pain of large adjustments and may be biologically advantageous, leading to faster bone formation, although this will require further study. Additionally, Orthospin's system sends real time updates to the physician's phone or computer, allowing him or her to monitor the patient's progress from anywhere. This limits the need for frequent clinic visits, potentially saving two or more visits throughout treatment [10]. The Orthospin struts are currently used with the Maxframe ${ }^{\mathrm{TM}}$ (Depuy Synthes) hexapod external fixation system and applied in the operating room like other struts. The patient therefore enjoys the benefits associated with modern external fixation treatment with added convenience and feasibility [10]. The Orthospin system is also entirely waterproof, and the robotic struts can be used to collect data regarding force experienced by a strut during each movement. This is expected to enhance our understanding of the biomechanics of gradual limb deformity correction and bone union.

\section{Early successful application}

Early applications of Orthospin technology in the United States have been promising. A 6-year-old patient presented with a 31 millimeter (mm) Limb Length Discrepancy (LLD) and 10 degrees of external tibial torsion (Figure 2A). At ten years old, his LLD worsened to $35 \mathrm{~mm}$ with a predicted LLD of $40 \mathrm{~mm}$, and he experienced 12 degrees of external tibial torsion (Figure $2 \mathrm{~B}$ and $2 \mathrm{C}$ ). At this point, his complex, multiplanar deformity (axial and rotational) was treated with osteoplasty of the tibia and fibula followed by the application of an external fixator equipped with Orthospin struts. Six days after the operation, the patient was prescribed a treatment plan with a $40 \mathrm{~mm}$ lengthening goal and a 12-degree rotation correction. One month after surgery, a distraction gap of $22 \mathrm{~mm}$ was achieved (Figure 3A). Two months after surgery, the full distraction gap of $40 \mathrm{~mm}$ was achieved, and the patient was given a residual adjustment schedule to correct 2 degrees of varus, $3 \mathrm{~mm}$ of medial translation, and $6 \mathrm{~mm}$ of anterior translation (Figure 3B). Ten days later, the patient presented for his final distraction visit and displayed no residual deformity (Figures 3C,4A). At this point the control box and motors were removed from the external fixator in the clinic to make the frame less bulky (Figure 4B). Three months after surgery, the patient returned to light, low impact physical activity (Figures 3D,5). Four months after surgery, the external fixator was removed (Figure 6). This case highlights the value and benefits associated with the Orthospin technology. The patient's multiplanar deformity was successfully corrected with an external fixator, but without the patient or family adjusting a single strut. The adjustments were well tolerated, and the patient reported no significant
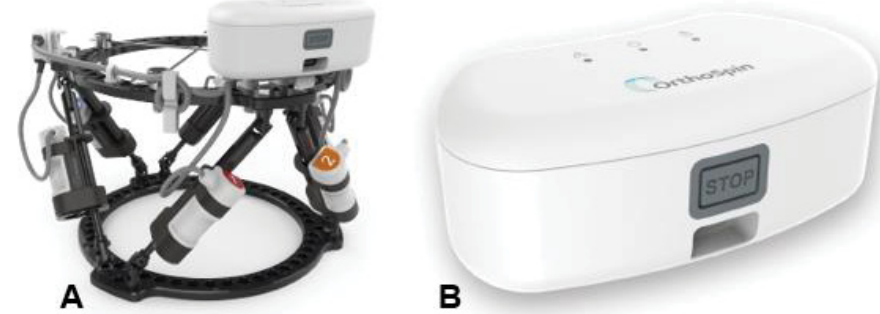

Figure 1: (A) Orthospin motorized "Smartstruts" and smart control box attached to an external fixator [12]. (B) Close-up of the smart control box [12].

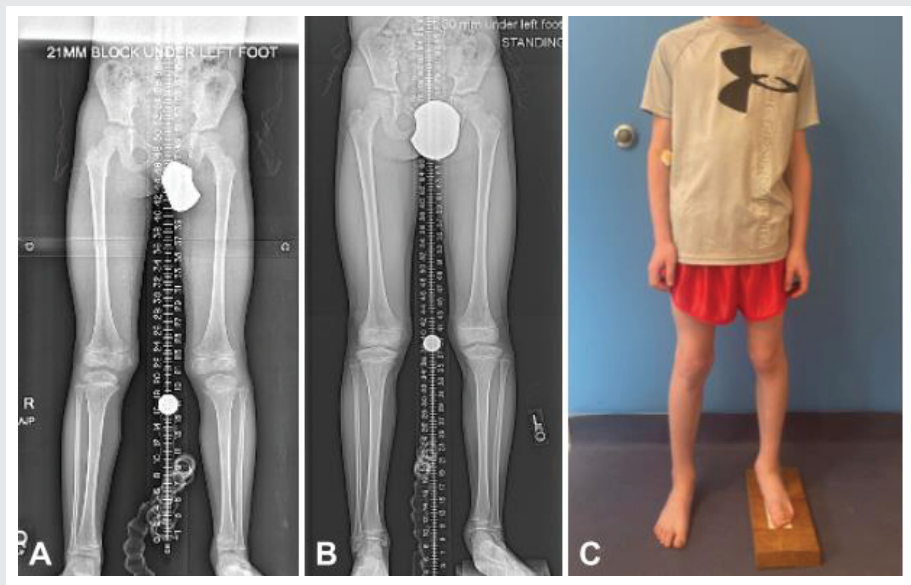

Figure 2: (A) X-Ray of patient at initial presentation with a $21 \mathrm{~mm}$ block under the left foot. (B) Preoperative X-Ray of patient with a $30 \mathrm{~mm}$ block under the left foot. (C) Preoperative clinical photo of patient showing $35 \mathrm{~mm}$ LLD. 
pain or need for additional medication throughout the process. The physician was able to track the patient's progress in realtime, so fewer clinic visits were needed than is typical for a large lengthening of four centimeters, saving the patient's family time and money. Overall, the patient underwent a less burdensome, less error-prone, and highly effective deformity correction. Minimal pain and need for narcotics were noted. The Orthospin technology only recently received FDA clearance, and the current small sample size presents a limitation to the study. However, the senior authors have successfully treated over 10 patients with the Orthospin system to date, and plan to continue to use the technology [13].
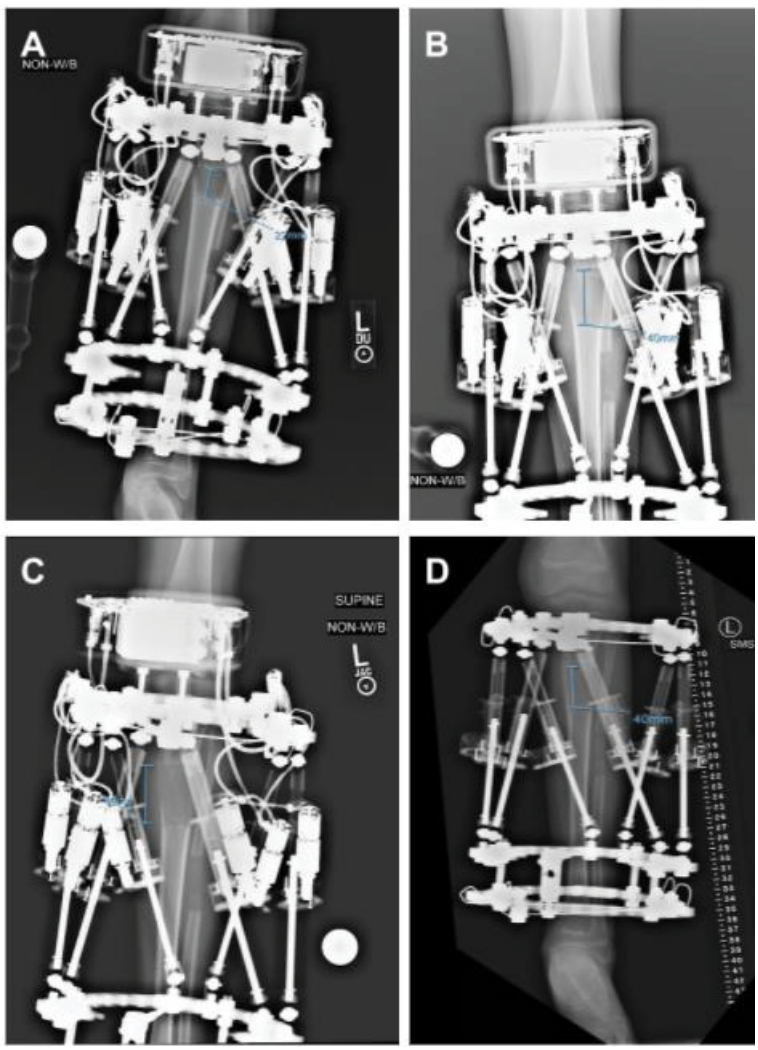

Figure 3: (A) 1-month postoperative $X$-Ray showing $22 \mathrm{~mm}$ distraction gap. Note the box on top of the frame controls the six struts below. (B) 2-month postoperative X-Ray showing full $40 \mathrm{~mm}$ distraction gap and initial bone regeneration. (C) X-Ray at final distraction visit showing full $40 \mathrm{~mm}$ distraction gap and elimination of residual deformities. (D) X-ray at date of return to light physical activity showing excellent bone regeneration.

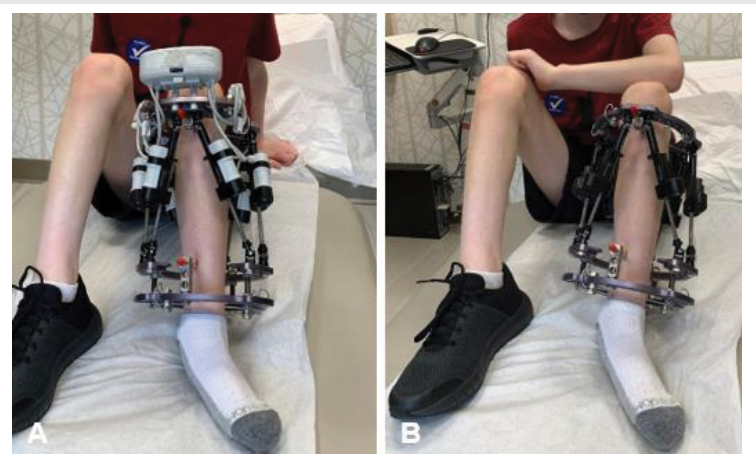

Figure 4: (A) Clinical photo at final distraction visit showing frame with Orthospin control box and motorized struts. (B) Clinical photo at final distraction visit showing frame after removal of control box and motors.
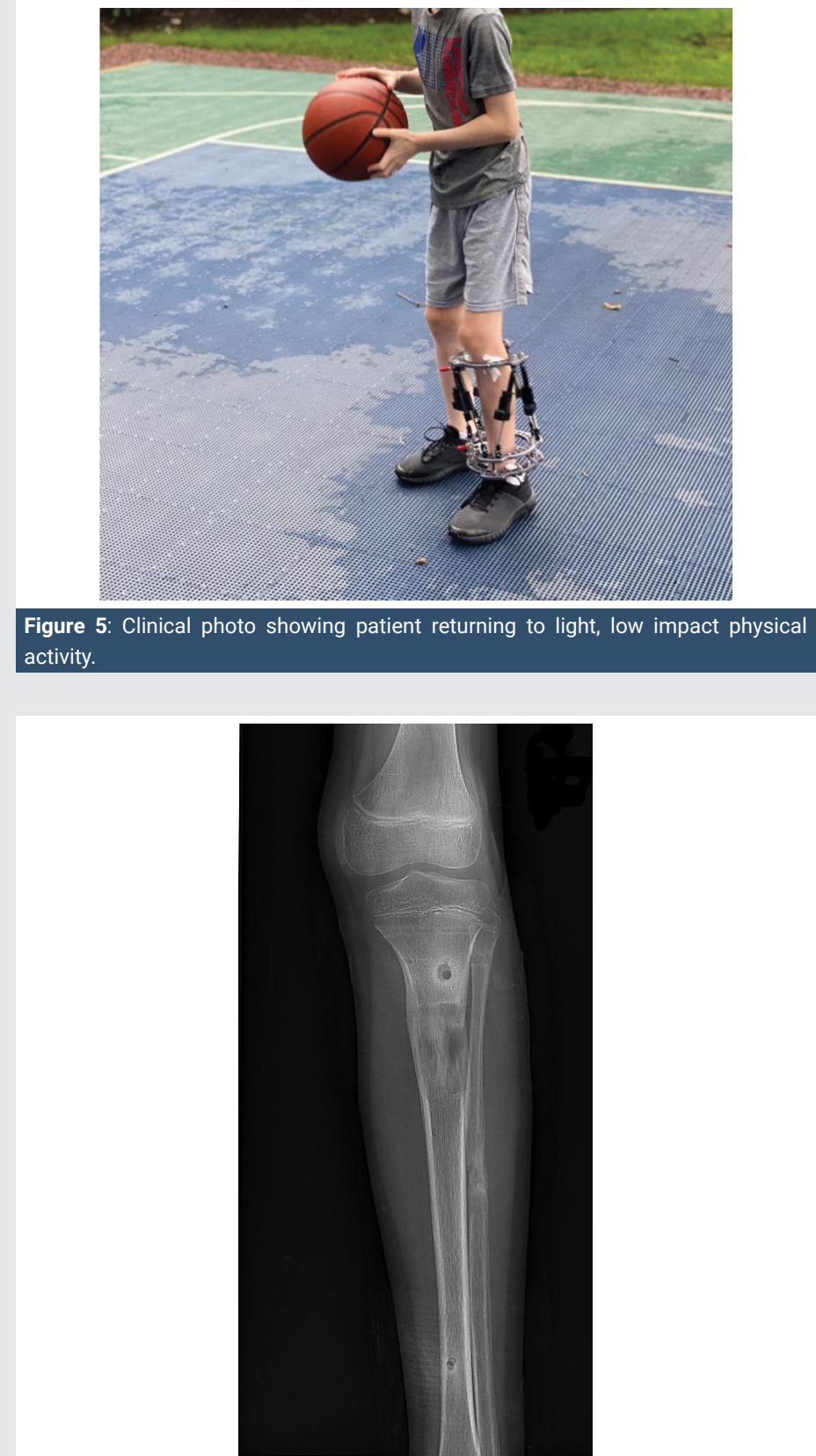

Figure 6: 4-month postoperative X-Ray after removal of the external fixator.

\section{Conclusion}

Modern external fixators represent a proven and often essential tool in the correction of multiplanar, complex deformities. Despite their remarkable versatility and effectiveness, they still suffer from flaws revolving around the need for manual adjustment of struts by patients and caregivers. Orthospin's Autostrut technology resolves these issues and improves the patient experience by automating the adjustment of struts and sending real-time feedback to doctors. The Orthospin system is FDA approved, and the global market for this technology is estimated at 800 million USD.

\section{References}

1. Hexapod External Fixator Deformity Correction (2021) posnacademy.org POSNAcademy. Link: https://bit.ly/2W1ubOq 
2. Principles of External Fixation (2014) Ota.org: Orthopaedic Trauma Association. Link: https://bit.ly/3sn9fx9

3. Fleming B, Paley D, Kristiansen T, Pope M (1989) A biomechanical analysis of the ilizarov external fixator. Clin Orthop Relat Res 95-105. Link: https://bit.ly/2VZup8A

4. Rozbruch SR, Fragomen AT, llizarov S (2006) Correction of tibial deformity with use of the ilizarov-taylor spatial frame. J Bone Joint Surg Am 88:156-174. Link: https://bit.ly/3AMLIbQ

5. What is the TAYLOR SPATIAL FRAME. smith-nephew.com: Smith \& Nephew. Link: https://bit.ly/3m6wxpX

6. Rozbruch SR, Pugsley JS, Fragomen AT, Ilizarov S (2008) Repair of tibial nonunions and bone defects with the taylor spatial frame. J Orthop Trauma 22: 88-95. Link: https://bit.ly/3snENTv

7. Rozbruch SR, Segal K, llizarov S, Fragomen AT, Ilizarov G (2010) Does the taylor spatial frame accurately correct tibial deformities? Clin Orthop Relat Res 468: 1352-1361. Link: https://bit.ly/3g619Gv
8. Horn DM, Fragomen AT, Rozbruch SR (2011) Supramalleolar osteotomy using circular external fixation with six-axis deformity correction of the distal tibia. Foot Ankle Int 32: 986-993. Link: https://bit.ly/3yXVZBm

9. OrthoSpin at Trendlines Medical Asia Road Show (2016) youtube.com: The Trendlines Group. Link: https://bit.ly/3CVhYez

10. OrthoSpin -- Smart External Fixation System (2016) youtube.com: The Trendlines Group. Link: https://bit.ly/3g8cBPw

11. OrthoSpin. trendlines.com: Trendlines. Link: https://bit.ly/37P2HxF

12. OrthoSpin. orthospin.com: Orthospin. Link: https://bit.ly/3CV8Zdt

13. Gigi R, Mor J, Lidor I, Ovadia D, Segev E (2021) Auto Strut: a novel smart robotic system for external fixation device for bone deformity correction, a preliminary experience. J Child Orthop 15(2):130-136. Link: https://bit.ly/3kmT6E3
Discover a bigger Impact and Visibility of your article publication with Peertechz Publications

\section{Highlights}

* Signatory publisher of ORCID

* Signatory Publisher of DORA (San Francisco Declaration on Research Assessment)

* Articles archived in worlds' renowned service providers such as Portico, CNKI, AGRIS, TDNet, Base (Bielefeld University Library), CrossRef, Scilit, J-Gate etc.

* Journals indexed in ICMJE, SHERPA/ROMEO, Google Scholar etc.

* OAI-PMH (Open Archives Initiative Protocol for Metadata Harvesting)

* Dedicated Editorial Board for every journa

* Accurate and rapid peer-review process

* Increased citations of published articles through promotions

* Reduced timeline for article publication

Submit your articles and experience a new surge in publication services (https://www.peertechz.com/submission).

Peertechz journals wishes everlasting success in your every endeavours.

Copyright: ( $) 2021$ Geffner AD, et al. This is an open-access article distributed under the terms of the Creative Commons Attribution License, which permits unrestricted use, distribution, and reproduction in any medium, provided the original author and source are credited.

Citation: Geffner AD, Reif TJ, Fragomen AT, Rozbruch SR (2021) Use of orthospin technology in the correction of complex limb deformities. Ann Robot Automation 5(1): 037-040. DOI: https://dx.doi.org/10.17352/ara.000012 